\title{
Políticas y modelos en Tinbergen y Muth
}

Policies and models Tinbergen and Muth

Javier Galán Figueroa*

\section{Resumen}

El presente documento revisa los trabajos de Jan Tinbergen y John Muth, quienes son considerados los padres de la teoría de la política económica y de las expectativas racionales, respectivamente. Se consideran sus trabajos a fin de retomar sus aportes sobre cómo los policimakers hacen política económica a partir de las preferencias y expectativas de los agentes en escenarios estáticos y dinámicos, permitiendo a las autoridades encontrar la mejor estrategia que permita alcanzar el máximo beneficio con el menor costo social.

Palabras clave:

- Política económica

- Expectativas racionales

\section{Abstract}

This paper reviews the work of Jan Tinbergen and John Muth who are the fathers of the economic policy and the hypothesis rational expectations respectively. Their work is used to show the main contributions on how policymakers design their economic policies based on the preferences and expectations of agents in static and dynamic environments to find the best strategy that maximizes the social benefit with the least cost.

\author{
Keywords: \\ - Economic Policy \\ - Rational Expectations
}

JEL: A2, B4

\section{Introducción}

El diseño de la política económica ha sido la tarea que más discusión ha generado en las diferentes escuelas de pensamiento que coexisten en la economía, cada una de ellas ha buscado determinar qué variables se deben considerar para encontrar un marco de referencia que les permita mantener un sistema económico que propicie el bienestar social. Para alcanzar este ideal, los economistas utilizan la racionalidad de los agentes como pieza clave para modelar las diferentes estrategias de política económica que la autoridad puede incurrir en períodos de certidumbre e incertidumbre.

Bajo esta línea, los trabajos de Jan Tinbergen y de John Muth son utilizados en el presente documento para retomar sus principales ideas sobre cómo las autoridades deben conducirse para llevar a cabo cualquier estrategia de política económica que les conduzca alcanzar el máximo beneficio social con el menor costo. De esta manera el documento se encuentra divido en dos secciones, en la primera sección se revisa la metodología de Jan Tinbergen sobre cómo se diseña un política óptima, mientras en la segunda se considera la propuesta de John Muth para mostrar la importancia de la racionalidad de los agentes en el diseño de las políticas. Por último se presenta algunos comentarios de tipo general.

* Profesor y Coordinador Académico de la Especialización en Economía Monetaria y Financiera, División de Estudios de Posgrado, Facultad de Economía, UnAM. " . " 


\section{El enfoque de Jan Tinbergen}

Desde los orígenes de la economía se ha discutido los mecanismos de cómo la ciencia económica debe actuar desde la perspectiva normativa y positiva para una eficiente asignación de los recursos escasos partiendo de los postulados del óptimo de Pareto o de los mercados competitivos tipo walrasiano. De esta manera los economistas han elaborado diferentes estrategias para garantizar el equilibrio entre los agentes económicos a través del tiempo con un costo mínimo a nivel social.

Mantener un equilibrio entre individuos no sólo garantiza una eficiente asignación de recursos sino que además, permite a la economía, como un sistema, funcione de acuerdo a las metas que la sociedad se ha planteado alcanzar en un determinado horizonte. En este aspecto, es donde los aspectos normativos y positivos de la economía interactúan entre sí para dar paso al proceso del diseño y conducción de la política económica, para ello es innegable partir de los trabajos que fueron desarrollados por Jan Tinbergen, el economista quien se dedicó a diseñar los mecanismos para que las autoridades puedan construir modelos capaces de conocer y explicar el estado actual de la economía. Así poder formular estrategias orientadas a elevar el bienestar social.

De acuerdo a Tinbergen (1952) los encargados de diseñar y conducir la política económica deben considerar en primera instancia los siguientes tres aspectos: a) determinar el interés general de la sociedad, b) seleccionar las políticas cuantitativas o cualitativas que conlleven a satisfacer el punto anterior, y c) seleccionar la teoría económica ad-hoc que les permita identificar las preferencias de los agentes y estas puedan ser consideradas en la formulación de la política óptima. Bajo estos aspectos, la política económica se define como el acto que describe el comportamiento económico de los hacedores de política, la cual incluye la teoría económica para interpretar las preferencias de los individuos y las empresas. En este sentido la política económica estará dirigida hacia la maximización de un conjunto de funciones que representan las preferencias de los agentes económicos y que el gobierno (o la autoridad) debe conducirla para alcanzar el bienestar social.

En lo referente al primer aspecto, la autoridad debe diseñar su política económica a partir del interés general de la sociedad, que puede ser la distribución del ingreso, bajo desempleo, la estabilización de precios, entre otros. Una vez que se ha definido el interés general la autoridad, la autoridad selecciona los instrumentos de política que serán utilizados para satisfacer las 
preferencias de la sociedad. En este sentido se define el conjunto de variables meta o variables target, $\Omega$, que representan el interés general de la sociedad, que a su vez está compuesto por los vectores las variables meta $y_{k}$, donde cada una de ellas hacen que $\Omega$ alcance un máximo.

En otras palabras, la autoridad selecciona aquellas variables meta que maximiza el bienestar general de la sociedad que es representada por $\Omega$. Una vez que se definen las variables target que maximizan el interés general de la sociedad, la autoridad selecciona los objetivos de política, los cuales operan como indicadores de corto plazo para conocer la distancia (o variabilidad) que se encuentra el valor observado con respecto con la variable meta. Para diferenciar entre una meta de un objetivo de política, Tinbergen (1952) señala que hay que distinguir, en primera instancia, entre lo que es una política cuantitativa de una cualitativa. Una política cualitativa refleja los aspectos cualitativos de la estructura económica que se estudia, mientras la cuantitativa muestra los parámetros (o los indicadores) de los instrumentos de política.

Lo anterior permite definir el principio de determinación de la política óptima si sólo las políticas cualitativas son consideradas posibles dentro de un conjunto de posibilidades o de alternativas. De esta manera los valores correspondientes para $\Omega$ son calculadas, mostrando alternativas sobre los valores más altos y las cuales podrían ser seleccionadas para el diseño de una determinada política. Para encontrar en qué punto $\Omega$ alcanza su máximo se considera el aspecto $\boldsymbol{c}$, donde la autoridad considera indicadores (o parámetros) para representar las preferencias individuales de los cuales podría implicar: i) fijar un indicador de preferencias colectivas, ii) la deducción del indicador a partir de las variables meta de la política económica, iii) selección adecuada de los instrumentos de las políticas cualitativas y cuantitativas, $\boldsymbol{i} \boldsymbol{v})$ determinación de los valores cuantitativos de las variables que representan los instrumentos que son seleccionados, y $\boldsymbol{v}$ ) la formulación de los conectores entre: $\boldsymbol{a}$ ) los valores de las metas y de los valores cuantitativos de las variables instrumento y $\boldsymbol{b}$ ) la estructura de la economía.

De acuerdo a Tinbergen los cinco puntos previos son interdependientes entre sí, debido a los instrumentos que son seleccionados no pueden estar separados de las metas y ambos no pueden estar separados de aquellas variables que se desempeñan como indicadores. Esta interdependencia permite conocer la estructura de la economía, sus restricciones, las variables meta, sus valores numéricos y la naturaleza de las variables que fungen como instrumento e indicador. Pero el problema para los policymakers consiste en encontrar los valores numéricos óptimos de los instrumentos que a su vez están en función 
a las variables meta, de la información disponible y de la interdependencia de todos los componentes que integran el modelo que se va utilizar para la política económica.

Existe un conjunto de variables que puede ser consideradas en el modelo de política económica, pero la autoridad debe clasificarlas de acuerdo a sus características y de esta manera saber que variables serán las metas, cuáles serán instrumentos, cuáles son las indicadoras o cuales son las variables que son consideradas como exógenas. En primera instancia, las variables que explican aquellos componentes que afectan a las variables metas y que no se encuentran incorporadas en el modelo, su influencia puede ser recogida por la variable residual, $u_{i}$.

En segunda instancia se encuentran las variables meta que pertenecen al conjunto de los intereses generales de la sociedad y también son las variables que garantizan el estado de equilibrio del sistema económico, por lo que estas también, están condicionadas a ciertas restricciones que la autoridad impone o desea alcanzar. El siguiente tipo de variables son los instrumentos de política, los cuales la autoridad las utiliza para afectar de manera directa a las variables objetivo y a las metas de acuerdo al marco analítico de la teoría económica, entre las variables que pueden ser instrumentos se encuentran los salarios, la tasa de interés nominal, el tipo de cambio, entre otras.

Una vez de haber clasificado las variables de acuerdo al interés de la autoridad se prosigue en la determinación de las relaciones estructurales que deben tener la característica de ser lineales para facilitar su solución mediante las técnicas de optimización. Una vez que se satisface este aspecto, la autoridad tendrá un modelo ad-hoc de política económica. Un aspecto que se debe considerar a la hora de determinar las relaciones estructurales es lo referente a que estas deben estar de acuerdo a la teoría económica, ya que esta última especificará en qué condiciones las variables serán meta, cuáles serán instrumentos y viceversa, de acuerdo a Tinbergen esto se le conoce como el problema de invertibilidad.

Si la autoridad cuenta ya con el modelo que le podría permitir alcanzar un máximo de $\Omega$ y por tanto maximizar el bienestar social, surge la siguiente cuestión ¿Qué tan efectiva es la política implementada? Una política económica es eficiente cuando el sistema de ecuaciones que componen el modelo alcanza a satisfacer los intereses generales de la sociedad. El principio del concepto de efectividad es un concepto cuantitativo que mide la tasa entre el efecto y el esfuerzo cuantitativo, es decir, suponiendo que se tiene un solo instrumento, $z$, con una sola variable meta $y$, entonces la medida cuantitativa será $d y / d z$, 
por lo que los valores de la variable estarán en función a los valores que tomen los parámetros de los instrumentos de política. Para el caso opuesto, el conjunto invertible los valores de las variables instrumento están ahora en función a los valores de las variables meta y su razón de medida se define como:

$$
\frac{1}{\partial z}
$$

Si la razón $\partial z / \partial y$ es grande, la efectividad de $\mathrm{z}$ con respecto a $y$ será pequeña. Esto implica que estas dos razones no son idénticas, ambas son funciones de los coeficientes estructurales pero no son parte de la misma función:

$$
\begin{aligned}
& \frac{\partial y}{\partial z}=\eta^{z} \\
& \frac{1}{\frac{\partial z}{\partial y}}=\frac{1}{\varsigma^{y}}
\end{aligned}
$$

Para seleccionar que tipo de función le conviene a la autoridad utilizar, se debe recurrir a la información estadística y ver el comportamiento de las variables esté acorde a la trayectoria convergencia de la variables meta y a los parámetros de los instrumentos de política, de esta manera conocer los valores esperados en los periodos subsecuentes. Por otro lado, la autoridad debe tener cuidado al seleccionar la función, ya que si recurre a la discrecionalidad, la política económica se volverá inconsistente y por tanto no se podrá maximizar el bienestar social ni se alcanzará el punto máximo donde se garantiza interés general.

En la conferencia titulada "The use of models: experience and prospects", que dio Jan Tinbergen al recibir el premio Nobel en economía 1969, enfatiza ante los miembros de la ciencia económica cómo los economistas que dedican su quehacer diario en el diseño y planeación de políticas económicas deben seguir una metodología en la construcción de modelos. En su opinión se debe considerar tres aspectos esenciales que todo modelo debe contener: primero, el economista o el policymaker debe elaborar una lista de las variables que habrán de considerarse; segundo, con base al primer punto, se debe elaborar una lista de ecuaciones o relaciones que deben obedecer las variables, y tercero, se debe verificar la validez de las ecuaciones, lo cual implica estimar los parámetros de las ecuaciones. 
A partir del tercer aspecto, el policymaker a su vez debe revisar el primer y segundo aspecto para garantizar que el error de estimación del modelo garantice su realismo para que esté acorde a la teoría que se utiliza. Lo anterior permite que el modelo construido pueda ser utilizado ya sea para fines de política, así como para dar soluciones a diversos problemas. Al utilizar este método trae consigo la ventaja de que los modelos se fundamentan en una determinada teoría económica, tomando en cuenta todos los fenómenos y relaciones pertinentes, por otra parte, permite confrontar el análisis estadístico de los datos observados con la realidad.

Otro aspecto que se hace énfasis sobre construcción de modelos es lo referente a que estos deben explicar el comportamiento de las políticas en el corto y largo plazo, a fin de analizar como los modelos son capaces de predecir el comportamiento de las variables meta (o target) de política. Para esto Tinbergen se refiere a los trabajos de Ragnar Frish (1933), también premio Nobel en economía 1969, quien introdujo los choques aleatorios a los modelos como elemento fundamental para explicar la dinámica interna de los sistemas económicos. Por otra parte, si bien, hay seguidores de la modelación económica quienes exageran en la construcción de modelos, según Tinbergen, esto se debe a que siguen modas con facilidad y estos son aquellos que no dominan las técnicas que suponen.

Para evitar las modas, es importante que los economistas, en especial los policymakers deben considerar tres puntos a seguir: el primero se refiere a la necesidad de introducir el aspecto espacio en los modelos de corte socioeconómico y de esta manera conocer los alcances de las políticas económicas. El segundo punto consiste en discutir que variables de corte social y político se pueden incorporar a los modelos económicos. El tercer punto hace referencia al uso de las técnicas de optimización, las cuales permiten al policymaker conocer las restricciones y las condiciones necesarias para que la política sea óptima. Este tercer aspecto, Tinbergen (1967) introduce los modelos econométricos dinámicos con la finalidad de verificar teorías con el fin de analizar tanto los ciclos económicos como las propiedades dinámicas que persisten a largo plazo, independientemente cualquiera que sea la condición inicial. La principal característica de estos modelos econométricos dinámicos que Tinbergen propuso son los multiplicadores de impacto que en los modelos var de Christopher Sims (1980) se han convertido como la herramienta fundamental de análisis de política económica.

Para ejemplificar lo anterior Tinbergen en sus libros "On the theory of economic policy" y "Economic policy: Principles and design" considera una estructura 
lineal de sistemas de ecuaciones para analizar la teoría de la política económica mediante un modelo lineal. Además se considera el caso donde sólo hay dos objetivos $\left(T_{1}, T_{2}\right)$ y dos instrumentos $\left(I_{1}, I_{2}\right)$, también se considera que los niveles deseados de $\left(T_{1}, T_{2}\right)$ están dados por los valores $\left(T_{1}^{*}, T_{2}^{*}\right)$. Entonces, cuando la economía opera a los niveles deseados, se dice que se encuentra en su punto óptimo que es equivalente al punto máximo de bienestar. Esta situación se deriva a partir de las ecuaciones siguientes:

$$
\begin{aligned}
& T_{1}=a_{1} I_{1}+a_{2} I_{2} \\
& T_{2}=b_{1} I_{1}+b_{2} I_{2}
\end{aligned}
$$

Donde cada objetivo se encuentra determinado por los mismos instrumentos, esta situación muestra que las autoridades pueden lograr los niveles deseados de ambos objetivos, siempre cuando, los instrumentos estén disponibles para las autoridades y cuyos efectos sobre los objetivos sean linealmente independientes entre sí, para que esto último ocurra, es necesario que $a_{1} / b_{1} \neq a_{2} / b_{2}$. Pero si ocurre que $a_{1} / b_{1}=a_{2} / b_{2}$, entonces sólo se alcanzará uno de los objetivos.

Para encontrar políticas óptimas, se sustituye los valores deseados $\left(T_{1}^{*}, T_{2}^{*}\right)$ en la ecuación (1) para obtener un sistema de ecuaciones de dos ecuaciones con dos incógnitas:

$$
\begin{aligned}
& T_{1}^{*}=a_{1} I_{1}+a_{2} I_{2} \\
& T_{2}^{*}=b_{1} I_{1}+b_{2} I_{2}
\end{aligned}
$$

Resolviendo el sistema de ecuaciones (2) para $\left(I_{1}, I_{2}\right)$ en términos de $\left(T_{1}^{*}, T_{2}^{*}\right)$, siempre cuando el determinante $\left(a_{1} b_{2}-a_{2} b_{1}\right) \neq 0$, se tiene

$$
\begin{aligned}
& I_{1}=\frac{\left(b_{2} T_{1}^{*}-a_{2} T_{2}^{*}\right)}{\left(a_{1} b_{2}-b_{1} a_{2}\right)} \\
& I_{2}=\frac{\left(a_{1} T_{2}^{*}-b_{1} T_{1}^{*}\right)}{\left(a_{1} b_{2}-b_{1} a_{2}\right)}
\end{aligned}
$$

además si se cumplen las condiciones de independencia lineal, la economía alcanza su punto óptimo $\left(T_{1}=T_{1}^{*}, T_{2}=T_{2}^{*}\right)$, por lo que estos dos instrumentos son independientes y suficientes para alcanzar los dos objetivos. Cabe mencionar que esto sólo ocurre cuando la economía opera bajo condiciones de linealidad, por lo que si la autoridad selecciona $\mathrm{M}$ metas que alcanzar, entonces 
tendrá que utilizar $\mathrm{N}$ instrumentos de política que sean linealmente independientes entre sí.

\section{La propuesta de John Muth}

Normalmente en los distintos manuales y libros de texto de economía se refieren a las expectativas como el factor que permite a las variables económicas tener cierto comportamiento temporal donde estas podrían estar sujetas a errores de predicción a través del tiempo. Entonces para explicar cómo las expectativas se forman y además se reflejen en el comportamiento optimizador (o racional) de los agentes se recurre al trabajo pionero de John Muth (1961) Rational Expectations and Theory of Price Movements, quién parte de la hipótesis de que los agentes de una economía no desperdician información y que las expectativas dependen generalmente de toda la estructura del sistema. Para contrastar su hipótesis, Muth desarrolla un análisis recursivo para determinar las condiciones dinámicas que permitan describir en comportamiento de los mercados de manera aislada, para ello describe el ejemplo de la especulación de las materias primas o commodities dentro del sistema económico.

Muth parte su estudio considerando que las trayectorias temporales de las variables económicas reflejan las fluctuaciones de los mercados, aunque esta conjetura sea limitada debido a que no se incluye el proceso de formación de las expectativas, entonces propone analizar el comportamiento de las variables mediante un modelo dinámico fundamentado en los trabajos de Arrow y Hurwicz (1958) y Arrow, Block y Hurwicz (1959) quienes explican las condiciones de estabilidad de los mercados competitivos walrasianos. El tipo de información es parte fundamental para Muth ya que considera que se debe utilizar datos adecuados y cómo estos pueden incorporarse en los modelos para poderlos estimar mediante procesos dinámicos, permitiendo con ello explicar el proceso de formación de las expectativas sobre los eventos del presente como del futuro. Incluso se puede realizar simulación sobre el comportamiento de las expectativas ante posibles escenarios o cambios en la estructura del modelo estimado previamente.

Por tanto, la hipótesis de las expectativas puede ser planteada considerando el caso de las empresas donde esperan que los niveles de producto para periodos futuros dependen de la distribución de la información y que esta se encuentra acorde a la teoría, aunque en este aspecto, la empresa podría estar sujeta a grandes errores que la propia teoría no puede explicar. En este sentido, esta hipótesis afirma tres aspectos: Primero, la información es escasa y 
el sistema económico en general no la desperdicia. Segundo, la formación de las expectativas depende en esencia de la estructura sobre el sistema o modelo que mejor describa el funcionamiento de la economía. Tercero, las predicciones públicas de los agentes podría no tener un efecto sustancial sobre el manejo de la economía.

Lo dicho previamente no afirma que los empresarios tengan un método de trabajo único que recoja la información suficiente para predecir de manera perfecta y que sus expectativas sean siempre las mismas. Para que las empresas puedan construir adecuadamente sus expectativas a partir del modelo construido se debe asumir los siguientes aspectos:

1. Los disturbios o componentes aleatorios se distribuyen como una distribución normal con media cero y varianza mínima, es decir:

$e_{t} \sim N\left(0, \sigma_{t}^{2}\right)$

2. Existe equivalentes ciertos para las variables para ser predecibles.

3. Las ecuaciones del sistema (o del modelo) deben incluir los valores esperados condicionados a las variables que son explicados fuera del sistema.

Cabe mencionar que estos supuestos no son fuertes por sí solos, sino que cada uno de ellos implica los demás. Para demostrar el proceso de formación de las expectativas se considera a continuación el siguiente modelo donde se asume las variaciones de los precios en el corto plazo en un mercado con un desfase fijo de la producción de un bien primario que no puede ser almacenado.

$$
\begin{aligned}
& C_{t}=-\beta p_{t} \\
& Q_{t}=\gamma p_{t}+u_{t} \\
& Q_{t}=C_{t}
\end{aligned}
$$

Las ecuaciones $(5 \mathrm{a})$ y $(5 \mathrm{~b})$ representan la demanda y la oferta respectivamente, mientras la ecuación $(5 \mathrm{c})$ indica la condición de equilibrio. Además, $Q_{t}$ representa el número de unidades producidas en un periodo y que es el mismo tiempo de vida que presenta la producción rezagada. $C_{t}$, es el monto de consumo del mercado, $p_{t}$, es el precio de mercado en el periodo t-esimo, $p_{t}^{e}$, es el precio esperado del mercado que prevalece durante el periodo $t$-esimo de acuerdo a la información disponible en los primeros $(t-1)$ periodos, $u_{t}$, es el 
componente aleatorio o error que representa las variaciones de la producción por factores aleatorios.

Por otra parte Muth utiliza las desviaciones de las variables desde su valor de equilibrio (o método de momentos alrededor de la media). De esta manera el sistema expresado en las ecuaciones (5a) y (5b) se obtiene la ecuación de los precios (6) donde el término no se conoce en el instante que se decide la producción pero si es conocido cuando el producto es comprado en el mercado.

$p_{t}=-\frac{\gamma}{\beta} p_{t}^{e}-\frac{1}{\beta} u_{t}$

La predicción del modelo consiste en encontrar y reemplazar en el periodo $t$ el término error por su valor esperado condicionado a los eventos pasados. Lo anterior se debe cumplir siempre cuando no exista correlación serial y además $E u_{t}=0$, de esta forma se obtiene.

$E p_{t}=-\frac{\gamma}{\beta} p_{t}^{e}$

Si la predicción de la teoría fue mejor que las expectativas de las empresas, entonces habría la oportunidad para la información privilegiada preveniente del conocimiento por la especulación de los inventarios, al operar una empresa o por la venta del servicio de pronósticos sobre el precio de un producto a las empresas. La oportunidad de ganancia podría no existir si la expectativa agregada de las empresas es la misma a la predicha por la teoría:

$$
E p_{t}=p_{t}^{e}
$$

Si en la ecuación (7) $\gamma / \beta \neq-1$ entonces el supuesto de la racionalidad de la ecuación (8) implica que $p_{t}^{e}=0$, o que el precio esperado es igual al precio del equilibrio. Mientras el término error se debe por la función de oferta, de los precios y por el movimiento de los precios de un periodo al siguiente a través de la curva de la demanda. Otro componente del término error son aquellos choques que no son predecibles pero con información a priori parte de este choque podría ser predecible de tal manera que la ecuación del precio esperado se define como: 
$p_{t}^{e}=-\frac{1}{\beta+\gamma} E u_{t}$

Pero si el choque es observable, entonces el valor de la esperanza condicional del modelo estimado podría ser encontrado directamente. Si el choque es no observable se debe obtenerse del pasado de las variables. En la situación donde las expectativas presentan correlación serial con el término error este último es una combinación lineal que depende de su pasado y se distribuye como una normal e independiente.

$$
u_{t}=\sum_{i=0}^{\infty} \omega_{i} \varepsilon_{t-i}, \quad E \varepsilon_{j}=0, \quad E \varepsilon_{i} \varepsilon_{j}=\left\{\begin{array}{l}
\sigma^{2} \rightarrow i=j \\
0 \rightarrow i \neq j
\end{array}\right.
$$

Bajo esta tesitura el precio es una función lineal donde el término error es también independiente:

$p_{t}=\sum_{i=0}^{\infty} W_{i} \varepsilon_{t-i}$

Donde $W_{i}$ son ponderadores. Entonces al incorporar al precio esperado la información de los primeros $(t-i)$ periodos se obtiene una expresión semejante a la ecuación (11) con la excepción de que $\varepsilon_{t}$ es reemplazado por su valor esperado, por tanto los precios esperados se escriben como:

$p_{t}^{e}=W_{0} E \varepsilon_{t}+\sum_{i=0}^{\infty} W_{i} \varepsilon_{t-i}=\sum_{i=0}^{\infty} W_{i} \varepsilon_{t-i}$

Si se considera ahora que $p_{t, L}$ es el precio esperado en el periodo $t+L$ que se obtiene mediante la información disponible en el periodo t-esimo, entonces la formula se convierte en:

$p_{t-L, L}=\sum_{i=L}^{\infty} W_{i} \varepsilon_{t-i}$ 
Sustituyendo la ecuación (13) en la ecuación (5b) se obtiene la siguiente condición de equilibrio de mercado:

$W_{0} \varepsilon_{t}+\left(1+\frac{\gamma}{\beta}\right) \sum_{i=1}^{\infty} W_{i} \varepsilon_{t-i}=-\frac{1}{\beta} \sum_{i=1}^{\infty} \omega_{i} \varepsilon_{t-i}$

Esta última ecuación es una identidad en la que el término $\varepsilon$ debe mantenerse para los valores observados de $\varepsilon_{j}$. Por lo tanto, los coeficientes de los correspondientes $\varepsilon_{j}$ en la ecuación deben ser iguales. Los ponderadores $W_{i}$ se definen a continuación:

$$
\begin{aligned}
& W_{0}=-\frac{1}{\beta} \omega_{0} \\
& W_{1}=-\frac{1}{\beta+\gamma} \omega_{i} ; \quad i=1,2,3, \cdots
\end{aligned}
$$

En las ecuaciones (15a) y (15b) los parámetros están dados por la relación entre precios y precios esperados en términos de la información pasada de los shocks. El problema consiste en mantener los resultados en términos de los valores históricos de las variables observables, en este sentido se desea encontrar la relación que cuente la siguiente estructura:

$$
p_{t}^{e}=\sum_{j=1}^{\infty} V_{j} p_{t-j}
$$

Resolviendo para los ponderadores $V_{j}$ en términos de los ponderadores $W_{j}$ mediante la sustitución de la ecuación (11) en la (12), lo que se obtiene:

$$
\sum_{i=1}^{\infty} W_{i} \varepsilon_{t-1}=\sum_{j=1}^{\infty} V_{j} \sum_{i=0}^{\infty} W_{i} \varepsilon_{t-i-j}=\sum_{i=1}^{\infty}\left(\sum_{j=1}^{i} V_{j} W_{i-j}\right) \varepsilon_{t-i}
$$

Donde la igualdad que debe mantener todos los choques y por tanto los coeficientes deben satisfacer es el siguiente sistema:

$$
W_{i}=\sum_{j=1}^{i} V_{j} W_{i-j} \quad i=1,2,3, \cdots
$$


Este sistema de ecuaciones presenta una estructura triangular, así que pondría resolverse de manera sucesiva para los coeficientes $V_{1}, V_{2}, V_{3}, \ldots$. Si los choques están distribuidos de manera independiente entonces $\omega_{0}=-1 / \beta$ y todos los demás serán cero, por lo que la ecuación (18) implicará:

$$
p_{t}^{e}=0
$$

$p_{t}=p_{t}^{e}+W_{0} \varepsilon_{t}=-\frac{1}{\beta} \varepsilon_{t}$

Los precios esperados estarán en función a los precios de equilibrio de cada periodo de tiempo.

\section{Comentarios finales}

En este documento se presentaron los enfoques de Jan Tinbergen y de John Munth, los cuales son piezas fundamentales para diseñar actualmente la política económica a fin de que la sociedad alcance su máximo bienestar, sin embargo en México se ha dejado de lado la formación del economista bajo este postulado, creando con ello una generación de economistas sin visión técnica y sin compromiso social. El desarrollo de las propuestas de Tinbergen y Muth vale la pena en sí mismo, pero sobre todo con la finalidad de mostrar que todo economista debe considerar las preferencias y las expectativas de los agentes para el diseño de cualquier política económica, para que al optimizarla trate de alcanzar el bienestar social. 


\section{Referencias}

Arrow, K. y L. Hurwicz (1958), "Stability of the gradient process in $n$-person games", Technical Report 67, Department of Economics, Stanford University, diciembre.

Arrow, Block y Hurwicz (1959), "On stability of the competitive equilibrium", Econometrica, vol. 27, núm. 1, enero.

Frisch, R. (1933), Propagation problems and impulse problems in dynamic economic, Oslo.

Muth, John. (1961), "Rational expectations and the theory of price movements", Econometrica, vol. 29, núm. 3, julio.

Tinbergen, Jan. (1952), On the theory of economic policy, North-Holland Publishing Company, Amsterdam.

(1967), Economic policy: principles and design, North-Holland Publishing Company, Amsterdam. 